\title{
ERRATUM: “QATAR-2: A K DWARF ORBITED BY A TRANSITING HOT JUPITER AND A MORE MASSIVE COMPANION IN AN OUTER ORBIT” (2012, ApJ, 750, 84)
}

\author{
Marta L. Bryan ${ }^{1}$, Khalid A. Alsubai ${ }^{2}$, David W. Latham ${ }^{3}$, Neil R. Parley ${ }^{4}$, Andrew Collier Cameron ${ }^{4}$, \\ Samuel N. Quinn ${ }^{3}$, Joshua A. Carter ${ }^{3}$, Benjamin J. Fulton ${ }^{5}$, Perry Berlind ${ }^{3}$, Warren R. Brown ${ }^{3}$, \\ Lars A. Buchhave ${ }^{6,7}$, Michael L. Calkins ${ }^{3}$, Gilbert A. Esquerdo ${ }^{3}$, Gábor Fúrész ${ }^{3,8}$, Uffe Gråe Jørgensen ${ }^{6,7}$, \\ Keith D. Horne ${ }^{4}$, Robert P. StefaniK ${ }^{3}$, Rachel A. Street ${ }^{5}$, Guillermo Torres ${ }^{3}$, Richard G. West ${ }^{9}$, Martin Dominik ${ }^{4,13}$, \\ Kennet B. W. Harpsøe ${ }^{6,7}$, Christine Liebig ${ }^{4}$, Sebastiano Calchi Novati ${ }^{10,11}$, Davide Ricci ${ }^{12}$, and Jesper F. Skottfelt ${ }^{6,7}$ \\ ${ }^{1}$ Department of Astronomy, Harvard University, Cambridge, MA 02138, USA \\ ${ }^{2}$ Qatar Foundation, P.O. Box 5825, Doha, Qatar \\ ${ }^{3}$ Harvard-Smithsonian Center for Astrophysics, 60 Garden Street, Cambridge, MA 02138, USA \\ ${ }^{4}$ SUPA, School of Physics and Astronomy, University of St Andrews, North Haugh, St Andrews, Fife KY16 9SS, UK \\ ${ }^{5}$ Las Cumbres Observatory Global Telescope Network, 6740 Cortona Drive, Suite 102, Goleta, CA 93117, USA \\ ${ }^{6}$ Niels Bohr Institute, University of Copenhagen, Juliane Maries vej 30, DK-2100 Copenhagen, Denmark \\ ${ }^{7}$ Centre for Star and Planet Formation, Geological Museum, Øster Voldgade 5, DK-1350 Copenhagen, Denmark \\ ${ }^{8}$ Konkoly Observatory of the Hungarian Academy of Sciences, Budapest, Hungary \\ ${ }^{9}$ Department of Physics and Astronomy, University of Leicester, Leicester LE1 7RH, UK \\ ${ }^{10}$ Dipartimento di Fisica “E.R. Caianiello," Universita degli Studi di Salerno, Via S. Allende, I-84081 Baronissi (SA), Italy \\ ${ }^{11}$ INFN, Gruppo Collegato di Salerno, Sezione di Napoli, Italy \\ 12 Institut d'Astrophysique et de Géophysique, Allée du 6 Août 17, Sart Tilman, Bât. B5c, B-4000 Liège, Belgium \\ Received 2013 December 11; published 2014 February 4
}

When the discovery paper was published, residuals from the 1.34 day orbital solution indicated a companion with several possible periods. With additional radial velocity measurements over the following months, the authors became concerned that the period of the outer planet was converging on one year. After checking for potential sources of error, the authors determined that there was an error in the barycentric correction, and that the outer planet was a detection of Earth's orbital motion. Qatar- 2 was the first target observed by TRES with negative declination, and this uncovered a bug in the code that parsed the sexigesimal declination-the minutes and seconds of arc were parsed as positive instead of negative. This resulted in a low amplitude modulation of the barycentric correction due to Earth's motion around the Sun. No other published result from TRES is affected by this error. A reanalysis using the correct barycentric velocity shows that the orbital solution for Qatar-2b is unchanged from the results published in the discovery paper.

\footnotetext{
${ }^{13}$ Royal Society University Research Fellow.
} 